\title{
Crystal Fibers for the LHCb Calorimeter Upgrade
}

\author{
Loris Martinazzoli ${ }^{(\mathbb{D}}$ \\ on behalf of the LHCb SPACAL R\&D group and Crystal Clear Collaboration
}

\begin{abstract}
The Large Hadron Collider beauty (LHCb) experiment is one of the four main particle detectors located at the Large Hadron Collider. After the high-luminosity upgrade of the accelerator, the expected radiation dose faced by the LHCb electromagnetic calorimeter will reach peak values of 1 MGy in the centermost part, an amount not tolerable by the currently employed Shashlik technology, which demands a novel radiation hard design. In addition, a time resolution of few tens of picoseconds and a cell lateral size of about $2 \mathrm{~cm}$ are planned for track reconstruction purposes by the collaboration. In this article, the crystal spaghetti design was evaluated, a sampling geometry where scintillating crystal fibers are embedded in the dense absorbing material. The prototype was tested at CERN super proton synchrotron (SPS) with muons and electrons measuring an energy resolution of $3 \%$ for electrons at $20 \mathrm{GeV}$, tilting the prototype by $3^{\circ}$ both horizontally and vertically with respect to the incident beam, and a time resolution of $79 \mathrm{ps}$. The results presented in this article show the feasibility of the design.
\end{abstract}

Index Terms-Calorimetry, fibers, garnets, high energy physics (HEP), scintillating crystals, spaghetti calorimeter (SPaCaL).

\section{INTRODUCTION}

$\mathbf{L}$ $\mathrm{HCb}$ is a single-arm forward particle spectrometer of the CERN Large Hadron Collider (LHC), investigating the physics of the $b$ quark and the parameters of charge conjugation parity symmetry (CP symmetry) violation [1]. Its current electromagnetic calorimeter employs the Shashlik technology, a sampling layout consisting of alternating scintillating plastic and lead tiles. However, after the highluminosity (HL) LHC upgrade in 2025, the calorimeter will face a considerable increase in the expected radiation dose, which requires investigating a series of new solutions; its inner part will require a completely new module design, implementing a highly radiation hard and dense material to sustain doses ranging in value from 200 up to 1 MGy while maintaining the present energy resolution $(2.2 \%$ for electrons at $20 \mathrm{GeV}$ [2]), whereas the rest will undergo a complete revision of the readout system. The aim is to provide the whole calorimeter with higher granularity and precise timing information to mitigate the occupancy, defined as the fraction of channels hit per bunch crossing, as well as reduce pileup, which will be of the utmost importance at future HL colliders [3]. To reach this goal, the cell size should be about $2 \mathrm{~cm}$ in

Manuscript received February 4, 2020; accepted February 11, 2020. Date of publication February 21, 2020; date of current version June 19, 2020. This work was supported by the European Union's Horizon 2020 Research and Innovation Programme through the Marie Skłodowska-Curie under Grant 644260 (Intelum).

The author is with CERN, 1211 Geneva, Switzerland, and also with the Dipartimento di Fisica "Giuseppe Occhialini," Università degli Studi di Milano-Bicocca, 20126 Milan, Italy (e-mail: loris.martinazzoli@cern.ch).

Color versions of one or more of the figures in this article are available online at http://ieeexplore.ieee.org.

Digital Object Identifier 10.1109/TNS.2020.2975570

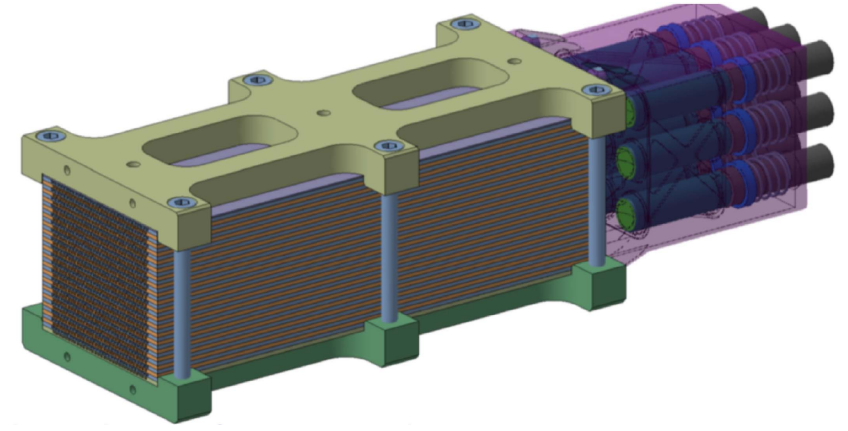

Fig. 1. Illustrative drawing of the spaghetti module, where the plates of absorbing material are stacked on top of each other and kept in place by a stainless steel frame. The readout is at the back and made of a matrix of nine photodetectors coupled to the absorber via light guides.

the inner region to combat the problems caused by overlapping clusters, while the time resolution is in the order 20-50 ps [4].

One of the possible solutions is the spaghetti calorimeter (SpaCal), a sampling design where scintillating fibers are embedded in an absorber made of dense material, e.g., lead or tungsten (Fig. 1). In such a geometry, the former will produce and transport the light to the photodetectors at the same time, effectively overcoming the need for wavelength-shifting (WLS) fibers that are the components of the shashlik design heavily affected by radiation damage [5]. On the other hand, the latter will keep the shower dimensions fairly reasonable, improving granularity. A SpaCal prototype was tested during this article, employing an absorber made of a W/Cu alloy and scintillating crystal garnets as active materials.

\section{MAterials AND Methods}

\section{A. Candidate Materials}

The candidate materials for this project were ceriumdoped crystal garnets, namely, $\mathrm{Gd}_{3} \mathrm{Al}_{2} \mathrm{Ga}_{3} \mathrm{O}_{12}$ : $\mathrm{Ce}$ (GAGG) and $\mathrm{Y}_{3} \mathrm{Al}_{5} \mathrm{O}_{12}$ : $\mathrm{Ce}$ (YAG). These materials were chosen because of their high light output and adequate timing properties for the detection of high-energy charged particles [6]. Moreover, radiation hardness of garnets and especially YAG, both bulk and in shape of fiber, was previously tested under high levels of gamma and proton irradiation, yielding excellent results [7]. The fibers employed for the following study were obtained from two different producers, Crytur for YAG and Fomos for GAGG, growing ingots through the Czochralski technique and subsequently cutting and polishing them to $1 \mathrm{~mm} \times 1 \mathrm{~mm} \times$ $100 \mathrm{~mm}$ fibers.

The radiation hardness of GAGG fibers was investigated at CERN proton synchrotron (PS) using the setup shown in Fig. 2. A GAGG fiber was exposed to a proton fluence of $3.5 \cdot 10^{15} \mathrm{~cm}^{-2}$ at $24 \mathrm{GeV}$, for a radiation dose of about 


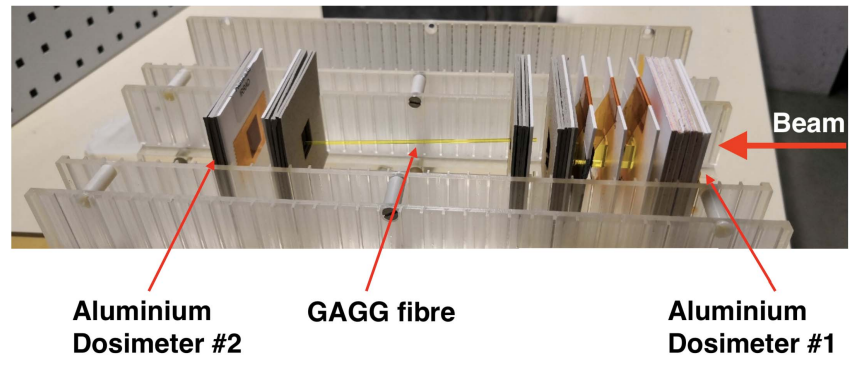

Fig. 2. Setup employed in the irradiation test at CERN PS. One GAGG fiber and three $1-\mathrm{cm}^{3}$ GAGG cubes (not discussed here) are placed between two aluminum dosimeters, one upstream, and the other downstream, employed to measure the radiation dose.

1.03 MGy. Before and after irradiation, the light attenuation of the fiber was measured. This was performed by exciting the fiber with 430-nm LEDs fixed at 1, 3, 5, 7, and $9 \mathrm{~cm}$ along its main axis and measuring at both ends the light output with Hamamatsu S1223-01 photodiodes. Two ClearPSM (Kuraray) optical fibers were initially placed instead of the photodiodes and plugged into an AvaSpec ULS2048 dualchannel spectrometer to verify that no light from the excitation source reached the readout; all the detected light lay in the range of the garnets emission, and no signal was present at about $430 \mathrm{~nm}$. Assuming a single-component exponential attenuation along the fiber axis $z$, which is a reasonable approximation at first order [8], the ratio $R(z)$ between the signals produced in the two photodetectors can be described by

$$
R(z)=e^{-2 \frac{z}{\lambda_{\text {att }}}}
$$

where the parameter $\lambda_{\text {att }}$ is the attenuation length of the fiber. Given the complexity in measuring transmission on a $1 \mathrm{~mm} \times 1 \mathrm{~mm}$ section, it was decided to quantify the radiation damage by means of this parameter, as in [7].

\section{B. SpaCal Prototype}

The spaghetti prototype tested in this article was composed of 39 plates $1.5 \mathrm{~mm} \times 60 \mathrm{~mm} \times 200 \mathrm{~mm}$ in dimension, made of a W/Cu alloy $(75 / 25 \%)$ with a density of $14.9 \mathrm{~g} / \mathrm{cm}^{3}$. Each plate had either 32 or 33 grooves of section $1.1 \mathrm{~mm} \times 1.1 \mathrm{~mm}$ carved on their surface, where the scintillating fibers were inserted. The plates were then stacked on top of each other, alternating the two groups to get a staggered layout of the fibers, and a stainless steel frame was employed to provide mechanical stability. The absorber plates were 25 radiation lengths $\left(X_{0}\right)$ deep.

Due to the constraints imposed by the maximum available ingots dimension, the crystal fibers were cut $100 \mathrm{~mm}$ long with a $1 \mathrm{~mm} \times 1 \mathrm{~mm}$ square section, whereas the plastic ones covered the whole length of the plates, which is $200 \mathrm{~mm}$. The prototype was divided into a front and a back section to match the crystals' length and each additionally into nine cells. The top and the middle cells were made of 13 plates each, the bottom ones, instead, only of 12 , leaving one row empty. This was put on the very top of the prototype. Each row was divided into three cells, the right and central ones comprising 11 grooves each, and the left one alternatively 10 or 11 , depending on the total

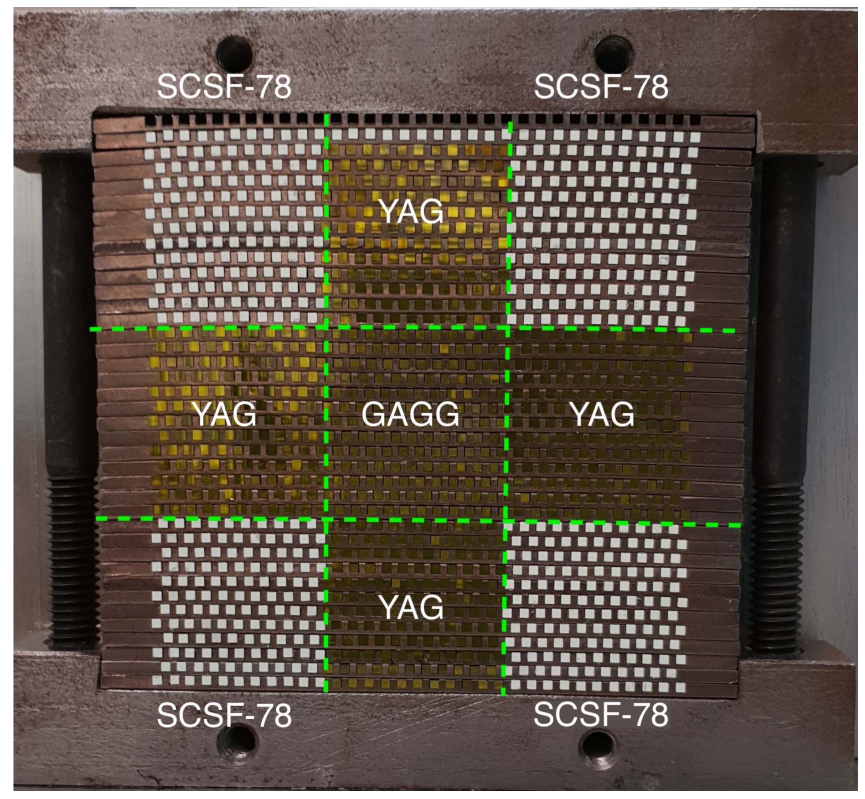

Fig. 3. Front picture of the spaghetti prototype. The green dotted lines ideally divide it into nine cells. The cells on the left have either 10 or 11 grooves per row, whereas the others have 11 . The bottom cells are comprised of 12 plates, whereas the central and the top ones are comprised of 13 .

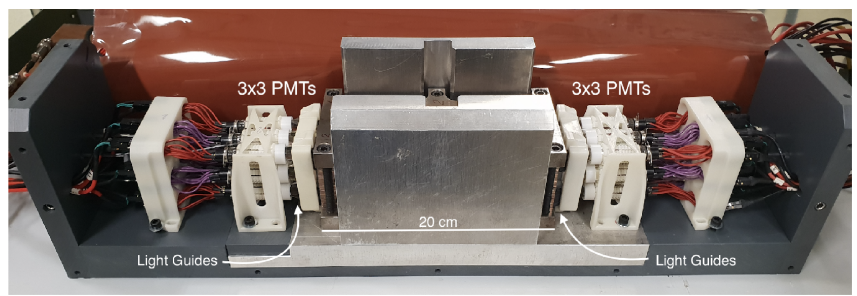

Fig. 4. Fully assembled SpaCal prototype as for this beam test. It is possible to see the frame (gray), which keeps straight the W/Cu plates, as well as the plastic holders (white) of the light guides, PMTs, and cables. In addition, plastic support (dark gray) was built to fix all the components in place, and a Kapton foil (brown) was employed to shield the prototype from the ambient light. The beam is entering the prototype from one of the two sides equipped with PMTs.

number of grooves per row, i.e., either 33 or 32 (see Fig. 3). Eight GAGG fibers were missing to completely fill the central cell in the back section. These were replaced by YAG fibers. For the same reason, the top row of the top central cell was filled with plastic fibers. The total number of fibers employed was 278 GAGG, 1096 YAG, and 548 SCSF-78 Kuraray plastic fibers.

To decouple front and back sections and extract more light from the cells filled with garnets, a thin layer of aluminum was deposited via chemical vapor deposition (CVD) on the fibers ends that were subsequently placed facing the center of the prototype. Each section was then additionally divided into nine cells, $20 \mathrm{~mm} \times 20 \mathrm{~mm}$ in size. In front of everyone (Fig. 4), a plexiglass light guide was positioned, shaped in a $20 \mathrm{~mm} \times 20 \mathrm{~mm} \times 30 \mathrm{~mm}$ truncated-pyramidal geometry with beveled edges, and dry coupled to a Hamamatsu R12421 photomultiplier tube (PMT). An optical fiber was placed close to each photodetector and connected to a single blue pulsed LED, employed for the measurement of the photoelectrons yield (see Section III-B). 


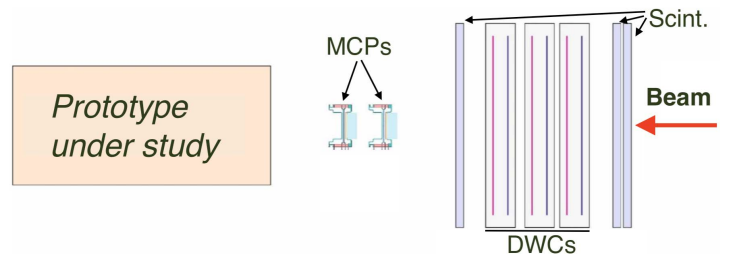

Fig. 5. Illustrative drawing of the beam test setup. There are two MCPs, three scintillating plates (Scint.) used in coincidence for triggering, and three DWCs.

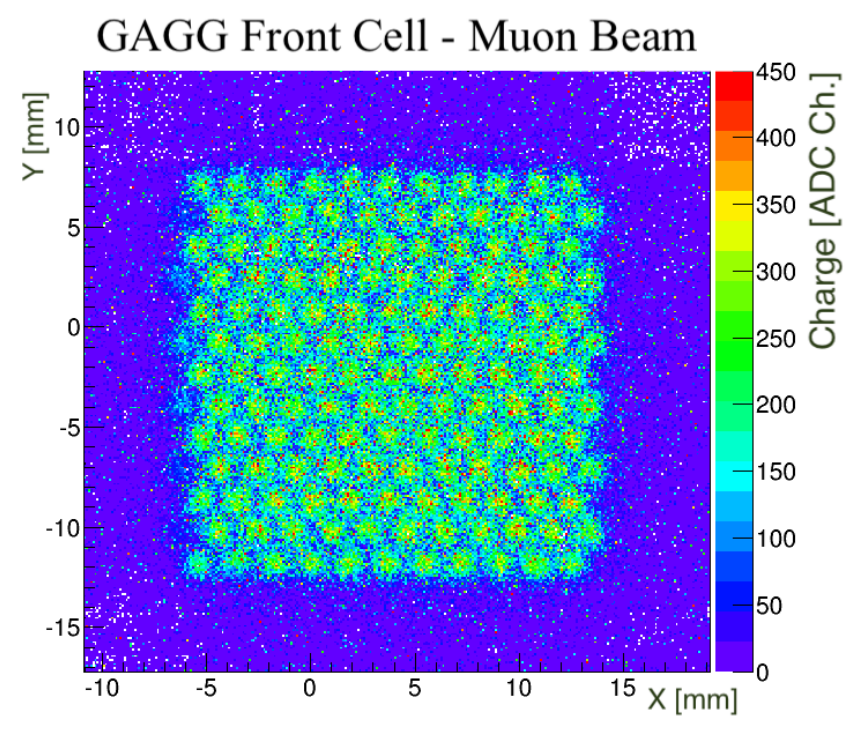

Fig. 6. Signal map for the front central cell generated by a muon beam parallel to the prototype main axis. The $X$ and $Y$ coordinates are given by the DWCs, whereas the color scale represents the energy deposited inside the prototype in ADC channels.

\section{Beam Test Setup}

The module described in Section II-B was tested at CERN super proton synchrotron (SPS) facilities with $180 \mathrm{GeV}$ muons and $20 \mathrm{GeV}$ electrons. The setup, shown in Fig. 5, consisted of three delay wire chambers (DWCs) for beam tracking and noise rejection, three scintillating plastic plates (Scint.) in coincidence for triggering, and two microchannel plate detectors (MCPs) for a time reference. The DWCs were read out through a CAEN TDC V1290N, whereas a LeCroy analogto-digital converter (ADC) 1182 performed charge integration of the 18 photodetectors, and a CAEN DT5742 was used to digitize the waveforms of the front central GAGG-filled cell for timing measurements.

\section{Calibration}

During the first phase of the beam test, a series of measurements was carried out exploiting minimum ionizing particles (MIPs), specifically muons at $180 \mathrm{GeV}$, and their straight trajectory. A full spatial characterization of the prototype response was carried out, as well as the intercalibration of the two longitudinal sections.

1) Muon Beam Parallel to the Prototype Axis: At first, the prototype was placed with its main axis parallel to the beam pipe in order to assess the uniformity of the response on

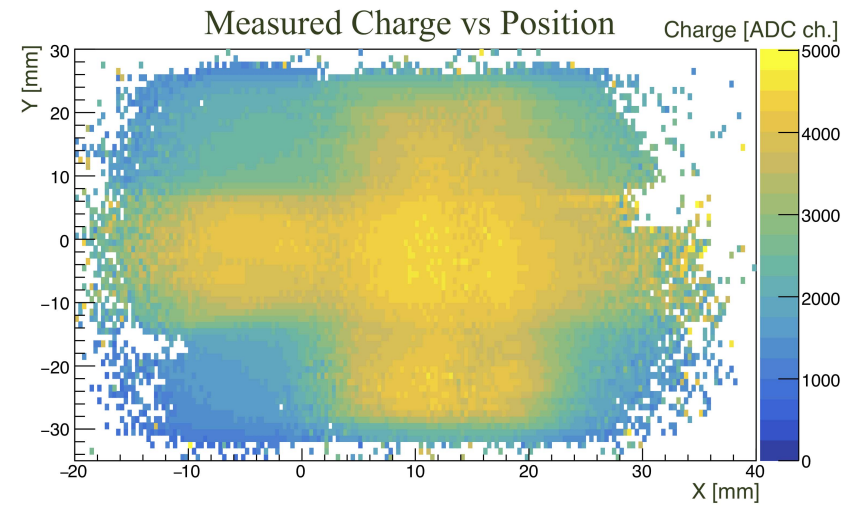

(a)

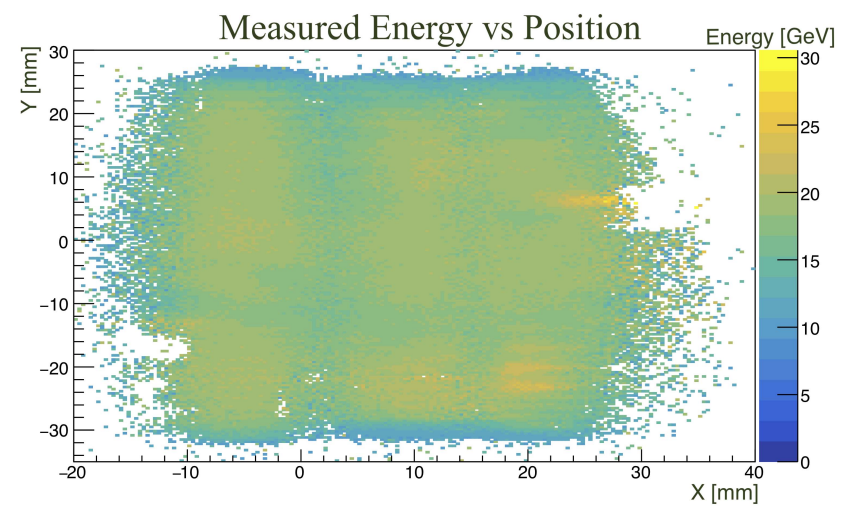

(b)

Fig. 7. Maps of the measured signal for the front cells (a) before and (b) after calibration, selecting only events above $10 \mathrm{GeV}$. The particle position is given by the DWCs. It can be seen how the differences in signal due to the various materials are straightened out with the calibration, giving a uniform map. The vertical stripes between the adjacent cells are due to the beam dimensions; multiple data acquisitions (DAQs) were done aiming the beam at the center of each cell; being the beam core dimensions $1.5 \mathrm{~cm} \times 2.5 \mathrm{~cm}$ on the $X$ and $Y$-axes, respectively, with a halo of impurities around, more than one cell were hit vertically at the same time, whereas only one horizontally, hence, the vertical stripes due to the lack of $20 \mathrm{GeV}$ events between the horizontally neighboring cells.

the orthogonal plane. In Fig. 6, the signal map for the front central GAGG cell is shown, where the coordinates of the particle are determined by the third DWC, the one closest to the prototype, giving a resolution on the particle impact point on the module of $0.3 \mathrm{~mm}$. Every single fiber is individually resolved in this way, and it is additionally possible to see a slight misalignment of the light guides; in fact, the dim signals on the left at $-6 \mathrm{~mm}$ on the $X$-axis can be attributed to YAG fibers from the neighboring cells. Such exercise highlights the great granularity of the spaghetti geometry.

2) Energy Calibration: The calibration process of the prototype was performed for each configuration in accordance with [9] and [10], minimizing deviations of the total measured energy $E_{i_{e v}}$ for all the events from the known electron beam energy $E_{0}=20 \mathrm{GeV}$

$$
\sum_{i_{e v}=0}^{N_{e v}}\left[E_{0}-E_{i_{e v}}\right]^{2}=\min
$$

where $i_{e v}$ is the $i-n$th event and $N_{e v}$ is the total number of events for the configuration in question. Only events in the 
Attenuation Length before and after Irradiation

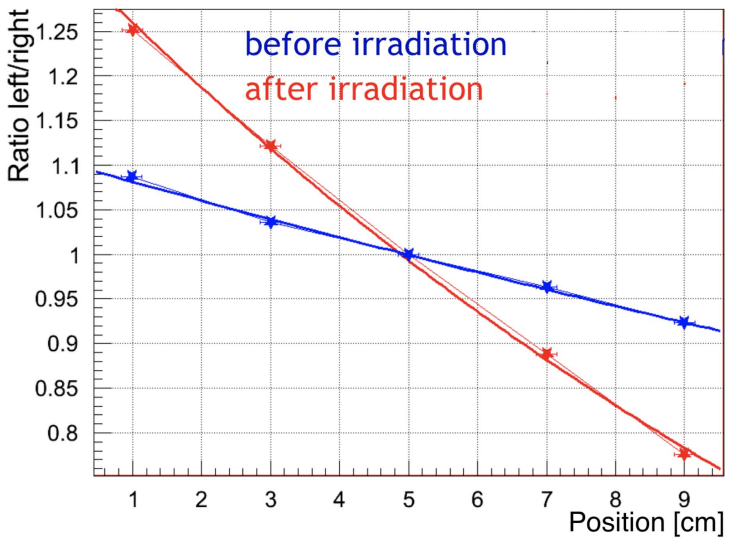

Fig. 8. Ratio between the signals of the two GAGG fiber ends measured before (blue) and after (red) irradiation. The fit functions are as in 1 .

TABLE I

Measured Attenuation Length of the GAGG Fiber BEFORE AND AFTER IRRADIATION

\begin{tabular}{lr}
\hline GAGG fibre & $\lambda_{a t t}[\mathrm{~cm}]$ \\
\hline Before irradiation & 101.5 \\
After irradiation & 33.6 \\
\hline
\end{tabular}

range $17-23 \mathrm{GeV}$ and hitting within a $4 \mathrm{~cm} \times 4 \mathrm{~cm}$ square around the center of the prototype were selected to reject impurities and avoid lateral leakages.

To avoid a dependency of the calibration factors on the beam energy, a series of acquisitions with $180 \mathrm{GeV}$ muon beams, which feature uniform energy deposition traversing the entire depth of the prototype, were performed just before and in the exact same configuration of each measurement with an electron beam. Intercalibrating the front and back sections with a uniform energy deposition, in fact, and keeping the ratio between the two sections fixed during the calibration process described earlier prevent introducing nonlinearities in the calibration factors [10].

The effects of the calibration process are shown in Fig. 7.

\section{RESULTS}

\section{A. Radiation Damage}

In the first place, the attenuation length of a GAGG fiber was measured before and after the irradiation discussed in Section II-A. The results are shown in Fig. 8 and reported in Table I. Although showing a nonnegligible drop, such attenuation lengths are still acceptable for radiation lengths below $1 \mathrm{~cm}$ as is the case for this prototype, therefore, establishing GAGG as a suitable material for the application from the point of view of radiation hardness.

\section{B. Photoelectrons Yield}

Once the detector was calibrated in energy, the photoelectrons yield could be measured. An optical fiber connected to a blue LED was placed close to each PMT, and periodically,
TABLE II

Photoelectrons Yield For EACH OF THE THREe MATERIALS EMPLOYED IN THE PROTOTYPE

\begin{tabular}{lcc}
\hline Material & Photoelectrons Yield $\left[\mathrm{MeV}^{-1}\right]$ & \pm \\
\hline GAGG & 9.71 & 0.22 \\
YAG & 6.76 & 0.16 \\
SCSF-78 & 1.15 & 0.14 \\
\hline
\end{tabular}

a light pulse was triggered. Measuring the mean and the variance of the signal produced by such events and exploiting Poisson statistics, the single-photoelectron signal $A_{\mathrm{ch}_{i}}^{1 \text { phel }}$ in the readout channel $\mathrm{ch}_{i}$ was calculated as

$$
A_{\mathrm{ch}_{i}}^{1 \mathrm{phel}}=\frac{\left\langle A_{L, \mathrm{ch}_{i}}\right\rangle}{\left\langle N_{L, \mathrm{ch}_{i}}\right\rangle}=\frac{\sigma_{L, \mathrm{ch}_{i}}^{2}}{\left\langle A_{L, \mathrm{ch}_{i}}\right\rangle}
$$

being $\left\langle A_{L, \mathrm{ch}_{i}}\right\rangle$ the average signal produced by the LED in the photodetector $\mathrm{ch}_{i}, \sigma_{L, \mathrm{ch}_{i}}^{2}$ its variance, and $\left\langle N_{L, \mathrm{ch}_{i}}\right\rangle$ the average numbers of photoelectrons produced by a LED pulse in each channel $\mathrm{ch}_{i}$. The conversion factor $C_{\mathrm{ch}_{i}}^{\text {phel }}$ from ADC channels to numbers of photoelectrons for the PMT $\mathrm{ch}_{i}$ was therefore

$$
C_{\mathrm{ch}_{i}}^{\mathrm{phel}}=\frac{1}{A_{\mathrm{ch}_{i}}^{1 \mathrm{phel}} .}
$$

The photoelectrons yield can be calculated computing the ratio between this latter calibration factor and the energy one and averaging all the channels filled with the same fiber material and all the measurements done. The results for each material are shown in Table II. The Monte Carlo simulations based on the GEANT4 toolkit [11] were employed to understand the light loss sources and the sampling fraction of the detector. These showed an average light collection efficiency of $2 \%$ and $2.3 \%$, not taking into consideration nonidealities of the fibers surface and the light guides-fibers coupling, which are difficult to accurately reproduce, and a sampling fraction of $19 \%$ and $14 \%$ for GAGG and YAG, respectively. On top of that, the quantum efficiency of the photodetector must be applied, which was estimated to be $8.6 \%$ and $8.9 \%$, convolving the quantum efficiency as in the datasheets and the radioluminescence spectra [12]. Such results are compatible with the measurements given in Table II.

\section{Light Attenuation Inside the Prototype}

The prototype was placed with its main axis orthogonal to the beam, and a longitudinal scan was performed to evaluate the light attenuation inside the fibers (Fig. 9). These attenuation curves display a double exponential behavior, with a short attenuation length of few $\mathrm{cm}$ and a long attenuation length in the range $80-100 \mathrm{~cm}$, quantitatively in accord with the values obtained during quality control tests. The former can be attributed to multiple causes [13], namely, skew rays, light rays originating off-axis, which travel in a long spiral path without crossing the fiber axis [14], Fresnel reflections, and the short-wavelength component of the radioluminescence spectra that tend to be more readily lost due to the materials' self-absorption. 
Light Attenuation along the Prototype's Longitudinal Axis

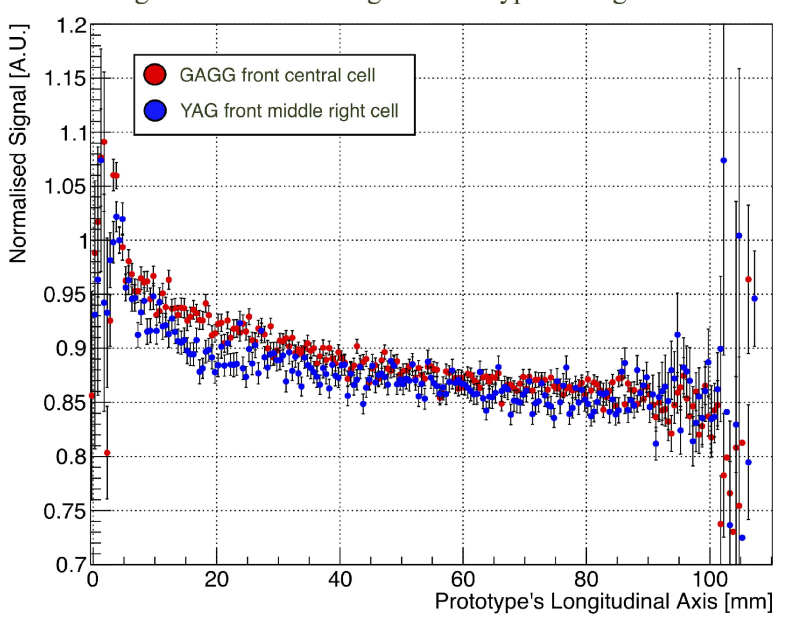

Fig. 9. Signal profiles generated by a $180-\mathrm{GeV}$ muon beam orthogonal to the prototype main axis for the GAGG front channel and the YAG front middle left channel. It is possible to see the two attenuation regimes: steeper in the first centimeters (close to the readout), and gentle afterward.

TABLE III

MEASURED ENERgy RESOLUTION FOR THE TwO CONFIgURATIONS TESTED

\begin{tabular}{cc}
\hline Tilt Angle $\left[{ }^{\circ}\right]$ & Energy Resolution $\sigma_{E} /\langle E\rangle[\%]$ \\
\hline $3 \oplus 0$ & 4.4 \\
$3 \oplus 3$ & 3.0 \\
\hline
\end{tabular}

\section{Energy Resolution}

A series of measurements with electron beams at $20 \mathrm{GeV}$ was performed. Fig. 6 shows how, in the case of particles at small incidence angles, the signal produced is heavily dependent on the entrance point at the prototype surface: an MIP hitting a fiber produces a clear signal, much stronger than hitting the absorber. Such a phenomenon, although mitigated by the transverse development of the cascade in the case of showering particles, greatly degrades energy resolution [15]. For this reason, it was decided to test the prototype in two configurations, tilting it with respect to the beam by $3^{\circ}$ in the horizontal plane (azimuthal angle, $3^{\circ} \oplus 0^{\circ}$ ) and by $3^{\circ}$ in both the horizontal and vertical planes (azimuthal and polar angles, $3^{\circ} \oplus 3^{\circ}$ ).

During the off-line analysis, a track selection was performed, and all the events with a signal too sparse between adjacent DWCs were rejected. The calculation of the energy resolution was done accepting events within a $10 \mathrm{~mm} \times 10 \mathrm{~mm}$ squared acceptance region in the center of the SpaCal to avoid transverse leakages introducing a detrimental low-energy tail while rejecting the events outside the $17-23 * \mathrm{GeV}$ energy interval. Both a direct computation of the standard deviation of the events in this latter interval and a Gaussian fit of the peak were performed. The average of the two was adopted as the final outcome and listed in Table III. The $3^{\circ} \oplus 3^{\circ}$ tilt angle configuration returns an energy resolution equal to $3 \%$ for electrons at $20 \mathrm{GeV}$ (Fig. 10), a value close to the targeted $2.2 \%$ of the current Large Hadron Collider beauty ( $\mathrm{LHCb}$ ) calorimeter modules.

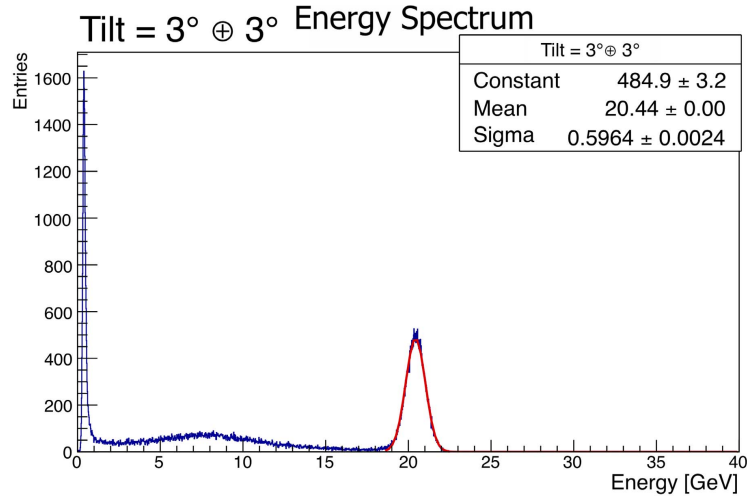

Fig. 10. Energy spectrum for the $3^{\circ} \oplus 3^{\circ}$ configuration and with a $10 \mathrm{~mm} \times$ $10 \mathrm{~mm}$ acceptance region before applying the $17-23-\mathrm{GeV}$ energy cut. The peak at $20 \mathrm{GeV}$ shows a non-Gaussian tail at low energies due to leakages. The entries are exclusive events labeled as the signal by the DAQ system and the analysis; therefore, pedestals are not present, and the beam impurities are evident at lower energies.

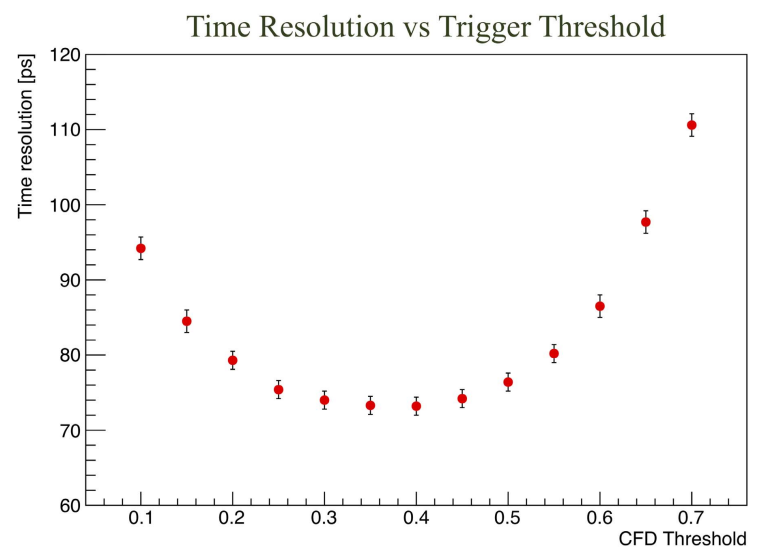

Fig. 11. Time resolution setting the trigger threshold at different fraction of the pulse maximum. The best value is $73.2 \pm 1.2$ ps obtained at $40 \%$.

\section{E. Time Resolution}

To assess the timing performances of the prototype, the CAEN DT5742 digitizer was connected to the photodetector of the front central GAGG cell, and the waveforms generated under excitation by a $20-\mathrm{GeV}$ electron beam incident at $0^{\circ}$ were recorded. This, combined with the information given by the two MCPs placed in front of the prototype, allowed to measure the time resolution of that cell. All the timestamps were obtained through constant fraction discriminator (CFD), whereas the reference time was defined as the average between the two MCPs' timestamps. The intrinsic resolution of the reference system was $21.8 \mathrm{ps}$, which was calculated by measuring the two MCPs one against the other with the electron beam during the test. The results, subtracting the reference resolution, for a PMT voltage bias of $730 \mathrm{~V}$ are shown in Fig. 11, and the best value is $73.2 \mathrm{ps} \pm 1.2 \mathrm{ps}$ standard deviation, reached setting the trigger threshold at $40 \%$ of the peak maximum. Such values prove to be close to the target range without optimization of any sort, and especially with a readout system positioned on the same side as the beam entering the module, enhancing the effect of longitudinal fluctuations, as well as a small incidence angle of the beam. 


\section{CONCLUSION}

This article was aimed at investigating the feasibility of the crystal spaghetti design for future calorimeters at $\mathrm{HL}$ colliders and, in particular, as a possible solution for the LHCb electromagnetic calorimeter upgrade.

At first, the radiation hardness of the candidate crystals was tested, finding a loss in attenuation length acceptable for a $1-M G y$ radiation dose in a $100-\mathrm{mm}$ GAGG fiber. Then, a 200-mm-long prototype was built and divided into two sets of $3 \times 3$ cells of $20 \mathrm{~mm} \times 20 \mathrm{~mm}$ section and $100-\mathrm{mm}$ long. The absorber was made of a W/Cu alloy and filled with $1 \mathrm{~mm} \times 1 \mathrm{~mm} \times 100 \mathrm{~mm}$ garnet crystal fibers, both GAGG and YAG, and 200-mm-long SCSF-78 scintillating plastic Kuraray fibers. It was tested at CERN SPS with electrons of $20 \mathrm{GeV}$, yielding an energy resolution up to $3 \%$ and a time resolution of 73.2 ps. Such results prove the validity of the spaghetti design and prompt a thorough optimization campaign, which will be carried out in the future.

\section{ACKNOWLEDGMENT}

The research leading to these results was conducted in the framework of the $\mathrm{LHCb}$ collaboration, the Crystal Clear Collaboration. The author would like to thank H. Gerwig and N. Siegrist from CERN for the design of the absorber, T. Schneider from CERN for the preparation of the fibers, and A. Barnyakov, Budker Institute of Nuclear Physics (BINP), Novosibirsk, for kindly providing the microchannel plate detector (MCP) photomultiplier tubes (PMTs).

\section{REFERENCES}

[1] R. Quagliani, “The LHCb detector at the LHC," J. Instrum., vol. 3, no. 8, 2008, S08005.

[2] A. Arefev et al., Beam Test Results of the LHCb Electromagnetic Calorimeter, doc. CERN-LHCb-2007-149, 2008.
[3] B. Schmidt, "The high-luminosity upgrade of the LHC: Physics and technology challenges for the accelerator and the experiments," J. Phys., Conf. Ser., vol. 706, May 2016, Art. no. 022002.

[4] Expression of Interest for a Phase-II LHCb Upgrade: Opportunities in Flavour Physics, and Beyond, in the HL-LHC Era, doc. CERN-LHCC2017-003, LHCb Collaboration, 2017.

[5] S. Barsuk et al., Radiation Damage of LHCb Electromagnetic Calorimeter, doc. LHCb 2000-033, 2000.

[6] M. T. Lucchini et al., "Timing capabilities of garnet crystals for detection of high energy charged particles," Nucl. Instrum. Methods Phys. Res. A, Accel., Spectrometers, Detectors Associated Equip., vol. 852, pp. 1-9, Apr. 2017.

[7] M. T. Lucchini, K. Pauwels, K. Blazek, S. Ochesanu, and E. Auffray, "Radiation tolerance of LuAG:Ce and YAG:Ce crystals under high levels of gamma- and proton-irradiation," IEEE Trans. Nucl. Sci., vol. 63, no. 2, pp. 586-590, Apr. 2016.

[8] K. Pauwels et al., "Single crystalline LuAG fibers for homogeneous dual-readout calorimeters," J. Instrum., vol. 8, no. 9, Sep. 2013, Art. no. P09019, doi: 10.1088/1748-0221/8/09/P09019.

[9] R. Wigmans and M. T. Zeyrek, "On the differences between calorimetric detection of electrons and photons," Nucl. Instrum. Methods Phys. Res. A, Accel. Spectrom. Detect. Assoc. Equip., vol. 485, pp. 385-398, 2002, doi: 10.1016/S0168-9002(01)02141-6.

[10] O. Ganel and R. Wigmans, "On the calibration of longitudinally segmented calorimeter systems," Nucl. Instrum. Methods Phys. Res. A, Accel. Spectrom. Detect. Assoc. Equip., vol. 409, nos. 1-3, pp. 621-628, May 1998.

[11] S. Agostinelli et al., "Geant4-A simulation toolkit," Nucl. Instrum. Methods Phys. Res. A, Accel. Spectrom. Detect. Assoc. Equip., vol. 506, pp. 250-303, 2003, doi: 10.1016/S0168-9002(03)01368-8.

[12] M. T. Lucchini et al., "Effect of $\mathrm{Mg}^{2+}$ ions co-doping on timing performance and radiation tolerance of cerium doped $\mathrm{Gd}_{3} \mathrm{Al}_{2} \mathrm{Ga}_{3} \mathrm{O}_{12}$ crystals," Nucl. Instrum. Methods Phys. Res. A, Accel. Spectrom. Detect. Assoc. Equip., vol. 816, pp. 176-183, Apr. 2016, doi: 10.1016/ j.nima.2016.02.004.

[13] G. F. Knoll, Radiation Detection and Measurement, 4th ed. Hoboken, NJ, USA: Wiley, 2010, sec. 8.3.

[14] J. D. Jackson, Classical Electrodynamics. New York, NY, USA: Wiley, 1999, sec. 8.10.

[15] D. Acosta et al., "Results of prototype studies for a spaghetti calorimeter," Nucl. Instrum. Methods Phys. Res. A, Accel. Spectrom. Detect. Assoc. Equip., vol. 294, pp. 193-210, Mar. 1990. 